\title{
A NOTE ON THE DIMENSION OF THE BIVARIATE SPLINE SPACE OVER THE MORGAN-SCOTT TRIANGULATION*
}

\author{
JIAN SONG DENG ${ }^{\dagger}$, YU YU FENG ${ }^{\dagger}$, AND JERNEJ KOZAK
}

Abstract. In [D. Diener, SIAM J. Numer. Anal., 27 (1990), pp. 543-551], a conjecture on the dimension of the bivariate spline space $S_{2 r}^{r}(\triangle)$ over the Morgan-Scott triangulation was posed. In this paper, it is proved that the conjecture should be modified for all even $r>2$.

Key words. bivariate spline space, Morgan-Scott triangulation, dimension

AMS subject classifications. 65D07, 41A63, 41A15

PII. S0036142998347772

1. Introduction. Let $S_{n}^{r}(\triangle)$ denote the space of $C^{r}$ differentiable bivariate piecewise polynomial functions of total degree $n$ over a regular triangulation $\triangle$. It is well known that in contrast to the univariate case, the problem of determining the dimension of $S_{n}^{r}(\triangle)$ is difficult. The lower and upper bound given in [10], [11] are far apart for large $r$. The lower bound actually gives the dimension of the spline space in many cases. The major difficulty is the fact that $\operatorname{dim} S_{n}^{r}(\triangle)$ generally depends on the geometric properties of the triangulation.

A case study for the dimension problem, encountered perhaps most frequently, is the Morgan-Scott triangulation $\triangle_{m s}$, i.e., the Schlegel diagram of an octahedron (Figure 1.1), introduced in [9]. The number of degrees of freedom in $S_{n}^{r}\left(\triangle_{m s}\right)$ and its dependence on the geometry of the partition have been studied in a number of papers including [6], [8], [12], [4], [5], [7]. In this paper we show that a conjecture on when the dimension exceeds the lower bound is correct for $r=2$ but is not correct for even $r \geq 4$.

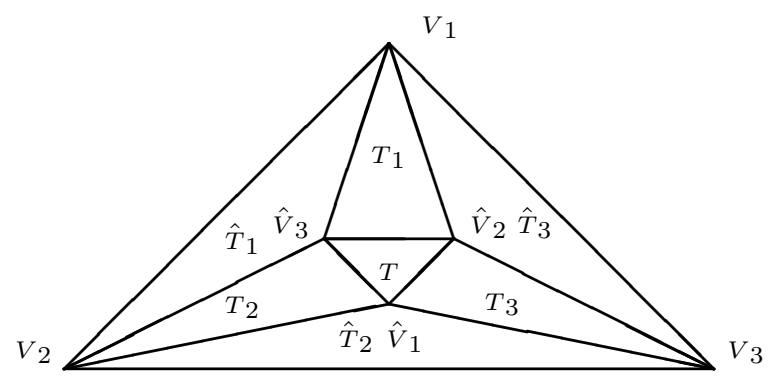

FIG. 1.1. The Morgan-Scott triangulation.

* Received by the editors November 19, 1998; accepted for publication (in revised form) June 30, 1999; published electronically March 6, 2000.

http://www.siam.org/journals/sinum/37-3/34777.html

${ }^{\dagger}$ Department of Mathematics, University of Science and Technology of China, Anhui, 230026 Hefei, People's Republic of China (dengjs@ustc.edu.cn, fengyy@ustc.edu.cn). The first and second authors were supported by the 973 project on Mathematical Mechanics, the National Natural Science Foundation, and the Science Foundation of the National Educational Committee of China.

${ }^{\ddagger}$ Department of Mathematics, University of Ljubljana, 1000 Ljubljana, Slovenija (Jernej.Kozak@ FMF.Uni-Lj.Si). This author was supported by the Ministry of Science and Technology of Slovenija. 
We follow the notation of [8], recalled on Figure 1.1. The boundary vertices are denoted by $V_{1}, V_{2}, V_{3}$, and interior vertices as $\hat{V}_{1}, \hat{V}_{2}, \hat{V}_{3}$. The triangulation $\triangle_{m s}$ consists of seven nondegenerate triangles $T, T_{i}, \hat{T}_{i}$. Note that the line through $\hat{V}_{i+1}$ and $\hat{V}_{i+2}$ separates the vertices $V_{i}$ and $\hat{V}_{i}$. Thus, if $r_{i}, s_{i}$ and $t_{i}$ are the barycentric coordinates of the vertex $V_{i}$ with respect to the triangle $T:=\hat{V}_{1} \hat{V}_{2} \hat{V}_{3}$,

$$
V_{i}=r_{i} \hat{V}_{i}+s_{i} \hat{V}_{i+1}+t_{i} \hat{V}_{i+2}, \quad i=1,2,3,
$$

the coordinate $r_{i}$ is negative. Here and throughout the paper, all the indexes, denoting the geometric objects in $\triangle_{m s}$ such as vertices, triangles, and barycentric coordinates are taken modulo 3, i.e., $V_{4}:=V_{1}, V_{-1}:=V_{2}$, etc. In order to avoid a discussion of a very particular case, let us further assume that no two adjacent edges are collinear. Then

$$
s_{i} \neq 0, t_{i} \neq 0, \quad i=1,2,3 .
$$

In [8], the case $n=2 r$ was studied, and the following bounds for the dimension $\operatorname{dim} S_{2 r}^{r}\left(\triangle_{m s}\right)$ were established:

$$
\alpha+\sigma \leq \operatorname{dim} S_{2 r}^{r}\left(\triangle_{m s}\right) \leq \alpha+\sigma+1,
$$

where

$$
\alpha:=\left(\begin{array}{c}
2 r+2 \\
2
\end{array}\right), \quad \sigma:=3 \sum_{j=1}^{r}(r+1-3 j)_{+}
$$

with $(\cdot)_{+}:=\max (\cdot, 0)$. Further, $\operatorname{dim} S_{2 r}^{r}\left(\triangle_{m s}\right)=\alpha+\sigma$ unless the barycentric coordinates of the vertices $V_{i}$ with respect to the triangle $T$ satisfy

$$
\begin{aligned}
& s_{1} s_{2} s_{3}=t_{1} t_{2} t_{3}, \quad r \text { odd, } \\
& s_{1} s_{2} s_{3}= \pm t_{1} t_{2} t_{3}, \quad r \text { even. }
\end{aligned}
$$

It was also conjectured in [8] that (1.2) gives the necessary and sufficient condition for

$$
\operatorname{dim} S_{2 r}^{r}\left(\triangle_{m s}\right)=\alpha+\sigma+1
$$

for all $r \geq 1$, and the conjecture was confirmed for $r=1,2$. The conjecture was further verified for $r=3$ in [5], but it turned out to be wrong for $r=4$ (see [7]). In this paper, we extend the last result to general $r$ and prove the following theorem.

TheOREM 1.1. Suppose that $s_{1} s_{2} s_{3}=-t_{1} t_{2} t_{3}$. For any even $r>2$,

$$
\operatorname{dim} S_{2 r}^{r}\left(\triangle_{m s}\right)=\alpha+\sigma .
$$

Thus the case $r=2$ is an exception, and the conjecture in [8] should be modified as follows: If $r$ is greater than 2, then $\operatorname{dim} S_{2 r}^{r}\left(\triangle_{m s}\right)=\alpha+\sigma+1$ if and only if

$$
s_{1} s_{2} s_{3}=t_{1} t_{2} t_{3} .
$$

2. Smoothness conditions. Smoothness conditions used in the paper will be expressed as a relation between the Bézier ordinates of the adjacent triangles as in [8]. Let

$$
\begin{aligned}
& P_{i, j, k}^{[\ell]}:=\frac{1}{2 r}\left(i V_{\ell}+j \hat{V}_{\ell+1}+k \hat{V}_{\ell+2}\right), \\
& \hat{P}_{i, j, k}^{[\ell]}:=\frac{1}{2 r}\left(i \hat{V}_{\ell-1}+j V_{\ell+1}+k V_{\ell}\right),
\end{aligned}
$$


be the domain points of triangles $T_{\ell}, \hat{T}_{\ell} \in \triangle_{m s}$ and $\beta_{i, j, k}^{[\ell]}, \hat{\beta}_{i, j, k}^{[\ell]}$ the corresponding Bézier ordinates associated with the domain points $P_{i, j, k}^{[\ell]}, \hat{P}_{i, j, k}^{[\ell]}$, respectively. Let $a_{i, j}, b_{i, j}$ and $c_{i, j}$ be the barycentric coordinates of $V_{j}$ with respect to the triangle $T_{i}$,

$$
V_{j}=a_{i, j} V_{i}+b_{i, j} \hat{V}_{i+1}+c_{i, j} \hat{V}_{i-1} .
$$

It is easy to derive the relations between $a_{i, j}, b_{i, j}, c_{i, j}$ and $r_{i}, s_{i}, t_{i}$. In particular,

$$
\begin{aligned}
& a_{\ell, \ell+1}=\frac{t_{\ell+1}}{r_{\ell}}, \quad b_{\ell, \ell+1}=r_{\ell+1}-\frac{s_{\ell} t_{\ell+1}}{r_{\ell}}, \quad c_{\ell, \ell+1}=s_{\ell+1}-\frac{t_{\ell} t_{\ell+1}}{r_{\ell}}, \\
& a_{\ell+1, \ell}=\frac{s_{\ell}}{r_{\ell+1}}, \quad b_{\ell+1, \ell}=t_{\ell}-\frac{s_{\ell} s_{\ell+1}}{r_{\ell+1}}, \quad c_{\ell+1, \ell}=r_{\ell}-\frac{s_{\ell} t_{\ell+1}}{r_{\ell+1}} .
\end{aligned}
$$

Given a spline $s \in S_{2 r}^{r}\left(\triangle_{m s}\right)$, let $p_{\ell}:=\left.s\right|_{T_{\ell}}$ and $\hat{p}_{\ell}:=\left.s\right|_{\hat{T}_{\ell}}$ denote the polynomial restrictions. In the Bernstein-Bézier form $p_{\ell}$ and $\hat{p}_{\ell}$ read as

$$
\begin{aligned}
& p_{\ell}(a, b, c)=\sum_{i+j+k=2 r} \beta_{i, j, k}^{[\ell]} \frac{(2 r) !}{i ! j ! k !} a^{i} b^{j} c^{k}, \\
& \hat{p}_{\ell}(\hat{a}, \hat{b}, \hat{c})=\sum_{i+j+k=2 r} \hat{\beta}_{i, j, k}^{[\ell]} \frac{(2 r) !}{i ! j ! k !} \hat{a}^{i} \hat{b}^{j} \hat{c}^{k},
\end{aligned}
$$

where $(a, b, c)$ and $(\hat{a}, \hat{b}, \hat{c})$ are barycentric coordinates with respect to the triangles $T_{\ell}$ and $\hat{T}_{\ell}$. If the ordinates corresponding to the domain points in the triangle $T$ are zero, then the smoothness conditions across the common edge of two adjacent triangles are simply

$$
\begin{aligned}
& \beta_{i, j, k}^{[\ell]}=0, \quad i \leq r, \\
& \hat{\beta}_{i, j, k}^{[\ell]}=0, \quad i \geq r,
\end{aligned}
$$

The remaining smoothness conditions [2], [3] can be written as

$$
\begin{aligned}
& \hat{\beta}_{i, j, k}^{[\ell]}=\left(a_{\ell, \ell+1} E_{1}+b_{\ell, \ell+1} E_{2}+c_{\ell, \ell+1} E_{3}\right)^{j} \beta_{k, 0, i}^{[\ell]}, \\
& \hat{\beta}_{i, j, k}^{[\ell]}=\left(a_{\ell+1, \ell} E_{1}+b_{\ell+1, \ell} E_{2}+c_{\ell+1, \ell} E_{3}\right)^{k} \beta_{j, i, 0}^{[\ell+1]},
\end{aligned}
$$

where $0 \leq i<r, \ell \leq j, k \leq r, i+j+k=2 r, \ell=1,2,3$, and $E_{1}, E_{2}, E_{3}$ are shift operators, defined in [3] as

$$
E_{1} f_{i, j, k}=f_{i+1, j, k}, \quad E_{2} f_{i, j, k}=f_{i, j+1, k}, \quad E_{3} f_{i, j, k}=f_{i, j, k+1} .
$$

Since for $1 \leq j, k \leq r, \hat{\beta}_{i, j, k}^{[\ell]}$ appears in both relations (2.1), the remaining smoothness conditions can be written as homogeneous relations among the Bézier ordinates for triangles $T_{1}, T_{2}, T_{3}$ only,

$$
\begin{aligned}
& \left(a_{\ell, \ell+1} E_{1}+b_{\ell, \ell+1} E_{2}+c_{\ell, \ell+1} E_{3}\right)^{j} \beta_{k, 0, i}^{[\ell]} \\
& \quad=\left(a_{\ell+1, \ell} E_{1}+b_{\ell+1, \ell} E_{2}+c_{\ell+1, \ell} E_{3}\right)^{k} \beta_{j, i, 0}^{[\ell+1]},
\end{aligned}
$$

where $i<r, 1 \leq j, k \leq r, i+j+k=2 r$, and $\ell=1,2,3$. 
Let $\mathcal{D}(\triangle)$ denote the set of the domain points for the triangulation $\triangle$. For any $t=P_{i, j, k}^{[\ell]} \in \mathcal{D}(\triangle)$, let $\lambda_{t}$ be the linear functional on $S_{n}^{r}\left(\triangle_{m s}\right)$ defined by $\lambda_{t} s:=\beta_{i, j, k}^{[\ell]}$. A set of the domain points $\mathcal{G} \subset \mathcal{D}(\triangle)$ is called the determining set for $S_{n}^{r}(\triangle)$ if for $s \in S_{n}^{r}(\triangle)$,

$$
\lambda_{t} s=0 \text { for all } t \in \mathcal{G} \Longrightarrow s=0 .
$$

If $\mathcal{G}$ is a determining set for $S_{n}^{r}(\triangle)$, then $\operatorname{dim} S_{n}^{r}(\triangle) \leq \# \mathcal{G}$, where $\# \mathcal{G}$ denotes the cardinality of the set of $\mathcal{G}$ (see [1]). We proceed to construct a particular determining set that will allow the conclusion of Theorem 1.1.

Let us follow the paper [8], and let $\mathcal{G}^{\prime}$ denote the set consisting of all domain points in the triangle $T$, the domain points

$$
P_{r+m, n-m, r-n}^{[\ell]}, 1 \leq n \leq \frac{r}{2}, 1 \leq m \leq(3 n-r-1)_{+}, \ell=1,2,3,
$$

and the domain points in the set

$$
\left\{P_{r+n-j, j, r-n}^{[\ell]}, \quad \frac{r}{2}<n<r, 1 \leq j \leq r-n, \ell=1,2,3\right\} \backslash\left\{P_{2 r-2,1,1}^{[\ell]}, \ell=1,2,3\right\} .
$$

As shown in [8], there is a total of $\alpha+\sigma-3$ domain points in $\mathcal{G}^{\prime}$. Let us assume that all Bézier ordinates associated with the domain points in $\mathcal{G}^{\prime}$ are zero. Then, as in the proof of Theorem 1 in [8], all Bézier ordinates $\beta_{i, j, k}^{[\ell]}$ with $i+j+k=2 r, i<2 r-2$, and $\ell=1,2,3$ and all $\beta_{2 r-2,2,0}^{[\ell]}$ and $\beta_{2 r-2,0,2}^{[\ell]}$ with $\ell=1,2,3$ are zero. There are only three index triples left to be examined. Put $A_{\ell}:=r a_{\ell+1, \ell} a_{\ell, \ell+1}-(r-1)$. The relations (2.1) for the index triple $(1, r-1, r)$, say for $\ell=2$, read

$$
\begin{aligned}
\hat{\beta}_{1, r-1, r}^{[2]} & =\left(a_{23} E_{1}+b_{23} E_{2}+c_{23} E_{3}\right)^{r-1} \beta_{r, 0,1}^{[2]} \\
& =a_{23}^{r-1} \beta_{2 r-1,0,1}^{[2]}+(r-1) a_{23}^{r-2} b_{23} \beta_{2 r-2,1,1}^{[2]}, \\
\hat{\beta}_{1, r-1, r}^{[2]} & =\left(a_{32} E_{1}+b_{32} E_{2}+c_{32} E_{3}\right)^{r} \beta_{r-1,1,0}^{[3]} \\
& =a_{32}^{r} \beta_{2 r-1,1,0}^{[3]}+r a_{32}^{r-1} c_{32} \beta_{2 r-2,1,1}^{[3]} ;
\end{aligned}
$$

hence

$$
\begin{aligned}
a_{23}^{r-1} \beta_{2 r-1,0,1}^{[2]} & -a_{32}^{r} \beta_{2 r-1,1,0}^{[3]} \\
& =r a_{32}^{r-1} c_{32} \beta_{2 r-2,1,1}^{[3]}-(r-1) a_{23}^{r-2} b_{23} \beta_{2 r-2,1,1}^{[2]} .
\end{aligned}
$$

Similarly at the point $(1, r, r-1)$,

$$
\begin{aligned}
a_{23}^{r} \beta_{2 r-1,0,1}^{[2]} & -a_{32}^{r-1} \beta_{2 r-1,1,0}^{[3]} \\
& =(r-1) a_{32}^{r-2} c_{32} \beta_{2 r-2,1,1}^{[3]}-r a_{23}^{r-1} b_{23} \beta_{2 r-2,1,1}^{[2]} .
\end{aligned}
$$

By combining (2.2) and (2.3) one obtains

$$
a_{23}^{r-1} \beta_{2 r-1,0,1}^{[3]}=a_{32}^{(r-1)} r_{2} \beta_{2 r-2,1,1}^{[3]}+r_{3} a_{23}^{r-2} A_{2} \beta_{2 r-2,1,1}^{[2]} .
$$

Thus for general $\ell$,

$$
\begin{aligned}
& a_{\ell, \ell+1}^{r-1} \beta_{2 r-1,0,1}^{[\ell]}=a_{\ell+1, \ell}^{r-1} r_{\ell} \beta_{2 r-2,1,1}^{[\ell+1]}+r_{\ell+1} a_{\ell, \ell+1}^{r-2} A_{\ell} \beta_{2 r-2,1,1}^{[\ell]}, \\
& a_{\ell+1, \ell}^{r-1} \beta_{2 r-1,0,1}^{[\ell+1]}=a_{\ell, \ell+1}^{r-1} r_{\ell+1} \beta_{2 r-2,1,1}^{[\ell]}+r_{\ell} a_{\ell+1, \ell}^{r-2} A_{\ell} \beta_{2 r-2,1,1}^{[\ell+1]} .
\end{aligned}
$$


Consider now the triple $(0, r, r)$, and take $\ell=2$ again. The relation $(2.1)$ gives

$$
\begin{aligned}
\hat{\beta}_{0, r, r}^{[2]}= & \left(a_{23} E_{1}+b_{23} E_{2}+c_{23} E_{3}\right)^{r} \beta_{r, 0,0}^{[2]} \\
= & a_{23}^{r} \beta_{2 r, 0,0}^{[2]}+r a_{23}^{r-1} b_{23} \beta_{2 r-1,1,0}^{[2]}+r a_{23}^{r-1} c_{23} \beta_{2 r-1,0,1}^{[2]} \\
& +r(r-1) a_{23}^{r-2} b_{23} c_{23} \beta_{2 r-2,1,1}^{[2]}, \\
\hat{\beta}_{0, r, r}^{[2]}= & \left(a_{32} E_{1}+b_{32} E_{2}+c_{32} E_{3}\right)^{r} \beta_{r, 0,0}^{[3]} \\
= & a_{32}^{r} \beta_{2 r, 0,0}^{[3]}+r a_{32}^{r-1} b_{32} \beta_{2 r-1,1,0}^{[3]}+r a_{32}^{r-1} c_{32} \beta_{2 r-1,0,1}^{[3]} \\
& +r(r-1) a_{32}^{r-2} b_{32} c_{32} \beta_{2 r-2,1,1}^{[3]},
\end{aligned}
$$

and generally

$$
\begin{array}{ll}
a_{\ell, \ell+1}^{r} \beta_{2 r, 0,0}^{[\ell]}+r a_{\ell, \ell+1}^{r-1} b_{\ell, \ell+1} \beta_{2 r-1,1,0}^{[\ell]}+r a_{\ell, \ell+1}^{r-1} c_{\ell, \ell+1} \beta_{2 r-1,0,1}^{[\ell]} & \\
\quad+r(r-1) a_{\ell, \ell+1}^{r-2} b_{\ell, \ell+1} c_{\ell, \ell+1} \beta_{2 r-2,1,1}^{[\ell]}-a_{\ell+1, \ell}^{r} \beta_{2 r, 0,0}^{[\ell+1]} & \\
\quad-r a_{\ell+1, \ell}^{r-1} b_{\ell+1, \ell} \beta_{2 r-1,1,0}^{[\ell+1]}-r a_{\ell+1, \ell}^{r-1} c_{\ell+1, \ell} \beta_{2 r-1,0,1}^{[\ell+1]} & =0 . \\
\quad-r(r-1) a_{\ell+1, \ell}^{r-2} b_{\ell+1, \ell} c_{\ell+1, \ell} \beta_{2 r-2,1,1}^{[\ell+1]} &
\end{array}
$$

Let

$$
\begin{aligned}
\beta:= & \beta_{2 r-2,1,1}^{[1]}, \beta_{2 r-2,1,1}^{[2]}, \beta_{2 r-2,1,1}^{[3]}, \beta_{2 r-1,0,1}^{[1]}, \beta_{2 r-1,1,0}^{[1]}, \beta_{2 r-1,0,1}^{[2]}, \\
& \left.\beta_{2 r-1,1,0}^{[2]}, \beta_{2 r-1,0,1}^{[3]}, \beta_{2 r-1,1,0}^{[3]}, \beta_{2 r, 0,0}^{[1]}, \beta_{2 r, 0,0}^{[2]}, \beta_{2 r, 0,0}^{[3]}\right)^{T}
\end{aligned}
$$

be a column vector with 12 components that combines all nonzero Bézier ordinates for our particular case. Each of the relations (2.4), (2.5), and (2.6) contributes three conditions; hence $\beta$ has to satisfy a homogeneous system of linear equations

$$
M \beta=0,
$$

where $M:=\left(m_{i j}\right)_{i, j=1}^{9 ; 12}$ is a $9 \times 12$ matrix, and its block representation reads

$$
M=\left(\begin{array}{lll}
M_{11} & M_{12} & M_{13} \\
M_{21} & M_{22} & M_{23}
\end{array}\right)
$$

where

$$
M_{11}=\left(\begin{array}{ccc}
-a_{12}^{r-2} r_{2} A_{1} & -a_{21}^{r-1} r_{1} & 0 \\
-a_{13}^{r-2} r_{3} A_{3} & 0 & -a_{31}^{r-1} r_{1} \\
0 & -a_{23}^{r-2} r_{3} A_{2} & -a_{32}^{r-1} r_{2} \\
-a_{12}^{r-1} r_{2} & -a_{21}^{r-2} r_{1} A_{1} & 0 \\
-a_{13}^{r-1} r_{3} & 0 & -a_{31}^{r-2} r_{1} A_{3} \\
0 & -a_{23}^{r-1} r_{3} & -a_{32}^{r-2} r_{2} A_{2}
\end{array}\right)
$$




$$
\begin{aligned}
& M_{12}=\operatorname{diag}\left(a_{12}^{r-1}, a_{13}^{r-1}, a_{23}^{r-1}, a_{21}^{r-1}, a_{31}^{r-1}, a_{32}^{r-1}\right), \\
& M_{21}=\left(\begin{array}{ccc}
r(r-1) a_{12}^{r-2} b_{12} c_{12} & -r(r-1) a_{21}^{r-2} b_{21} c_{21} & 0 \\
0 & r(r-1) a_{23}^{r-2} b_{23} c_{23} & -r(r-1) a_{32}^{r-2} b_{32} c_{32} \\
-r(r-1) a_{13}^{r-2} b_{13} c_{13} & 0 & r(r-1) a_{31}^{r-2} b_{31} c_{31}
\end{array}\right), \\
& M_{22}=\left(\begin{array}{cccccc}
r a_{12}^{r-1} c_{12} & r a_{12}^{r-1} b_{12} & -r a_{21}^{r-1} c_{21} & -r a_{21}^{r-1} b_{21} & 0 & 0 \\
0 & 0 & r a_{23}^{r-1} c_{23} & r a_{23}^{r-1} b_{23} & -r a_{32}^{r-1} c_{32} & -r a_{32}^{r-1} b_{32} \\
-r a_{13}^{r-1} c_{13} & -r a_{13}^{r-1} b_{13} & 0 & 0 & r a_{31}^{r-1} c_{31} & r a_{31}^{r-1} b_{31}
\end{array}\right) \\
& M_{23}=\left(\begin{array}{ccc}
a_{12}^{r} & -a_{21}^{r} & 0 \\
0 & a_{23}^{r} & -a_{32}^{r} \\
-a_{13}^{r} & 0 & a_{31}^{r}
\end{array}\right),
\end{aligned}
$$

and $M_{13}$ is a $6 \times 3$ zero matrix.

3. Two lemmas. In this section, we prove two lemmas.

Lemma 3.1. Let $s_{1} s_{2} s_{3}=-t_{1} t_{2} t_{3}$. Then the relations

$$
r_{1} r_{2}+s_{1} t_{2}=0, \quad r_{2} r_{3}+s_{2} t_{3}=0, \quad r_{3} r_{1}+s_{3} t_{1}=0
$$

cannot hold all at the same time.

Proof. Let $s_{1} s_{2} s_{3}=-t_{1} t_{2} t_{3}$, and suppose that all relations in (3.1) hold. Since $r_{i}, s_{i}, t_{i}$ are barycentric coordinates, $r_{i}+s_{i}+t_{i}=1$, and

$$
1=\left(r_{1}+s_{1}+t_{1}\right)\left(r_{2}+s_{2}+t_{2}\right)\left(r_{3}+s_{3}+t_{3}\right) .
$$

Expand the right side of this equation and omit the terms that sum to 0 . This produces

$$
\begin{aligned}
1= & \left(r_{1}+s_{1}+t_{1}\right)\left(r_{2}+s_{2}+t_{2}\right)\left(r_{3}+s_{3}+t_{3}\right) \\
= & r_{1} r_{2} r_{3}+r_{1}\left(s_{2}+t_{2}\right)\left(s_{3}+t_{3}\right) \\
& +r_{2}\left(s_{3}+t_{3}\right)\left(s_{1}+t_{1}\right)+r_{3}\left(s_{1}+t_{1}\right)\left(s_{2}+t_{2}\right) \\
& +s_{1}\left(r_{2} r_{3}+s_{2} t_{3}\right)+t_{1}\left(r_{2} r_{3}+s_{2} t_{3}\right)+s_{2}\left(r_{1} r_{3}+s_{3} t_{1}\right)+t_{2}\left(r_{1} r_{3}+s_{3} t_{1}\right) \\
& +s_{3}\left(r_{1} r_{2}+s_{1} t_{2}\right)+t_{3}\left(r_{1} r_{2}+s_{1} t_{2}\right)+\left(s_{1} s_{2} s_{3}+t_{1} t_{2} t_{3}\right) \\
= & r_{1} r_{2} r_{3}+r_{1}\left(s_{2}+t_{2}\right)\left(s_{3}+t_{3}\right) \\
& +r_{2}\left(s_{3}+t_{3}\right)\left(s_{1}+t_{1}\right)+r_{3}\left(s_{1}+t_{1}\right)\left(s_{2}+t_{2}\right) \\
= & r_{1} r_{2} r_{3}+r_{1}\left(1-r_{2}\right)\left(1-r_{3}\right)+r_{2}\left(1-r_{3}\right)\left(1-r_{1}\right)+r_{3}\left(1-r_{1}\right)\left(1-r_{2}\right)<0,
\end{aligned}
$$

since all $r_{i}$ are negative. This contradiction proves the lemma.

Lemma 3.2. Let $M=\left(M_{i j}\right)_{i, j=1}^{2 ; 3}=\left(m_{i j}\right)_{i, j=1}^{9 ; 12}$ be the matrix given in (2.8). Then $\operatorname{rank} M=8$ if and only if $s_{1} s_{2} s_{3}=t_{1} t_{2} t_{3}$. Otherwise the rank of $M$ is 9 .

Proof. Assumption (1.1) implies $\operatorname{det}\left(M_{12}\right) \neq 0$. If additionally $s_{1} s_{2} s_{3} \neq \pm t_{1} t_{2} t_{3}$, then

$$
\operatorname{det}\left(M_{23}\right)=\frac{\left(t_{1} t_{2} t_{3}\right)^{r}-\left(s_{1} s_{2} s_{3}\right)^{r}}{\left(r_{1} r_{2} r_{3}\right)^{r}} \neq 0
$$


and the rank of $M$ is clearly 9 since the last 9 columns of $M$ are linearly independent. In order to compute rank $M$ in the case $s_{1} s_{2} s_{3}= \pm t_{1} t_{2} t_{3}$, several row and column rank-preserving transformations on $M$ will be carried out. We shall describe only the sequence of the necessary operations and shall not write out the consecutive matrices obtained in the process. All these intermediate matrices will be denoted by the same letter $M$, in order to avoid notational complications; thus we shall think of $M$ as an empty cupboard whose shelves are filled with different items in each turn. The sequence of the operations to be performed on $M$ is as follows:

1. Multiply the second block column by $M_{12}^{-1}$. This reduces $M_{12}$ to the $6 \times 6$ identity matrix.

2. Subtract column $2 j+2$ multiplied by $(r-1) a_{j, j+1}^{r-2} b_{j, j+1}$ and column $2 j+3$ multiplied by $(r-1) a_{j, j+2}^{r-2} c_{j, j+2}$ from column $j, j=1,2,3$. Replace row 9 by

$$
\text { row } 7 \times s_{2}^{r} s_{3}^{r}+\text { row } 8 \times t_{1}^{r} t_{2}^{r}+\text { row } 9 \times s_{2}^{r} t_{2}^{r} .
$$

Since $s_{1} s_{2} s_{3}= \pm t_{1} t_{2} t_{3}$, the entries $m_{9 j}$ for $j=1,2,3,10,11,12$ are now reduced to zero.

3. Eliminate all the entries in the matrix $M_{11}$, i.e., substract

$$
\left(\begin{array}{l}
M_{12} \\
M_{22}
\end{array}\right) M_{11}
$$

from the first block column. This replaces each element $m_{9 j}$ for $j=1,2,3$ by the sum $\sum_{k=1}^{6} m_{k j} m_{9, k+3}$. In the case $j=1$, the new value of the entry $m_{91}$ turns out to be

$$
\begin{array}{r}
m_{91}=-r \frac{t_{2}^{r-1}}{r_{1}^{r-1} s_{1}^{r-1}}\left(s_{2}\left(-2 s_{1}^{r} s_{2}^{r} s_{3}^{r}+s_{1}^{r-1} s_{2}^{r-1} s_{3}^{r-1} t_{1} t_{2} t_{3}+t_{1}^{r} t_{2}^{r} t_{3}^{r}\right)\right. \\
\left.+r_{2} r_{3} t_{1} t_{2}\left(s_{1}^{r-1} s_{2}^{r-1} s_{3}^{r-1}-t_{1}^{r-1} t_{2}^{r-1} t_{3}^{r-1}\right)\right) .
\end{array}
$$

Similar expressions are obtained for $m_{92}$ and $m_{93}$.

Since now $M_{11}=\emptyset, M_{12}=I$, and $M_{13}=\emptyset$, as well as the first and the second row of $M_{23}$ are by (1.1) linearly independent, the first eight rows of $M$ are linearly independent. But the third row of $M_{23}$ is trivial, hence the matrix $M$ will be of full rank if and only if the last row of $M_{21}$ is not trivial. Suppose that $r$ is even. If $s_{1} s_{2} s_{3}=-t_{1} t_{2} t_{3}$, the entries $m_{91}, m_{92}$, and $m_{93}$ get simplified to

$$
\begin{aligned}
& m_{91}=2 r \frac{s_{1} s_{2}^{r} s_{3}^{r} t_{2}^{r-1}}{t_{3} r_{1}^{r-1}}\left(r_{2} r_{3}+s_{2} t_{3}\right), \\
& m_{92}=2 r \frac{s_{1}^{r} s_{2} s_{3}^{r} t_{3}^{r-1}}{t_{1} r_{2}^{r-1}}\left(r_{3} r_{1}+s_{3} t_{1}\right), \\
& m_{93}=2 r \frac{s_{1}^{r} s_{2}^{r} s_{3} t^{r-1}}{t_{2} r_{3}^{r-1}}\left(r_{1} r_{2}+s_{1} t_{2}\right) .
\end{aligned}
$$

Lemma 3.1 then implies that $m_{91}, m_{92}$, and $m_{93}$ are not all zero. Hence the rank of $M$ is 9 . On the other hand, if $s_{1} s_{2} s_{3}=t_{1} t_{2} t_{3}$, then $m_{91}=m_{92}=m_{93}=0$ and $\operatorname{rank} M=8$. 
4. The proof of the theorem. Suppose that $\beta_{2 r, 0,0}^{[1]}$ and any two of

$$
\beta_{2 r-2,1,1}^{[\ell]}, \ell=1,2,3,
$$

are zero; for example, the first two. Then, by Lemma 3.2, the system of linear equations (2.7) has only the trivial solution if $s_{1} s_{2} s_{3}=-t_{1} t_{2} t_{3}$. Therefore, the set

$$
\mathcal{G}:=\mathcal{G}^{\prime} \cup\left\{P_{2 r, 0,0}^{[1]}, P_{2 r-2,1,1}^{[1]}, P_{2 r-2,1,1}^{[2]}\right\}
$$

is the determining set for $S_{2 r}^{r}\left(\triangle_{m s}\right)$. There is a total of $\alpha+\sigma$ domain points in $\mathcal{G}$. Thus $\operatorname{dim} S_{2 r}^{r}\left(\triangle_{m s}\right) \leq \alpha+\sigma$. But in [10], [11] the expression $\alpha+\sigma$ is proved to be a lower bound for $\operatorname{dim} S_{2 r}^{r}\left(\triangle_{m s}\right)$, so the equality

$$
\operatorname{dim} S_{2 r}^{r}\left(\triangle_{m s}\right)=\alpha+\sigma
$$

must hold. The proof of Theorem 1.1 is completed.

\section{REFERENCES}

[1] P. Alfeld, And L. L. Schumaker, The dimension of bivariate spline spaces of smoothness $r$ for degree $d \geq 4 r+1$, Constr. Approx., 3 (1987), pp. 189-197.

[2] W. Böhm, G. Farin, and J. Kahmann, A survey of curve and surface method in CAGD, Comput. Aided Geom. Design, 1 (1984), pp. 1-60.

[3] G. Z. Chang, And P. J. Davis, The convexity of Bernstein polynomials over triangle, J. Approx. Theory, 40 (1984), pp. 11-28.

[4] Z. B. Chen, Y. Y. Feng, And J. Kozak, The blossom approach to the dimension of the bivariate spline space, J. Comput. Math., to appear.

[5] Z. B. Chen, J. S. Deng, and Y. Y. Feng, On the dimension of the $C^{3}$ spline space for Morgan-Scott partition, J. USTC, 28 (1998), pp. 505-512 (in Chinese).

[6] Y. S. ChOU, L. Y. Su, AND R. H. WANG, The dimension of bivariate spline over triangulations, in Multivariate Approximation Theory III, ISLAM 75, W. Schempp and K. Zeller, eds., Birkhäuser-Verlag, Basel, 1985, pp. 71-83.

[7] J. S. Deng, M. C. XI, AND Y. Y. FEnG, On the dimension of the $C^{4}$ spline space for MorganScott partition, Appl. Math. J. Chinese Univ., Ser. A, 13 (1998), pp. 91-98 (in Chinese).

[8] D. Diener, Instability in the dimension of spaces of bivariate piecewise polynomials of degree $2 r$ and smoothness order $r$, SIAM J. Numer. Anal., 27 (1990), pp. 543-551.

[9] J. Morgan and R. Scott, The Dimension of $C^{1}$ Piecewise Polynomials, manuscript, 1977.

[10] L. L. Schumaker, Lower bounds for the dimension of the spaces of piecewise polynomials in two variables, in Multivariate Approximation Theory, W. Schempp and K. Zeller, eds., Birkhäuser-Verlag, Basel, 1979, pp. 396-412.

[11] L. L. Schumaker, Bounds on the dimension of spaces of multivariate piecewise polynomials, Rocky Mountain J. Math., 14 (1984), pp. 251-264.

[12] X. Q. SHI, The singularity of Morgan-Scott triangulation, Comput. Aided Geom. Design, 8 (1991), pp. 201-206. 